\title{
On the vector-valued Littlewood-Paley-Rubio de Francia inequality
}

\author{
Denis Potapov, Fedor Sukochev and Quanhua Xu
}

\begin{abstract}
The paper studies Banach spaces satisfying the LittlewoodPaley-Rubio de Francia property $\mathrm{LPR}_{p}, 2 \leq p<\infty$. The paper shows that every Banach lattice whose 2-concavification is a UMD Banach lattice has this property. The paper also shows that every space having $\mathrm{LPR}_{q}$ also has $\mathrm{LPR}_{p}$ with $q \leq p<\infty$.
\end{abstract}

\section{Introduction}

Let $X$ be a Banach space and let $L^{p}(\mathbb{R} ; X)$ be the Bochner space of $p$-integrable $X$-valued functions on $\mathbb{R}$. If $X=\mathbb{C}$, we abbreviate $L^{p}(\mathbb{R} ; X)=L^{p}(\mathbb{R}), 1 \leq p<\infty$. For every $f \in L^{1}(\mathbb{R} ; X), \hat{f}$ stands for the Fourier transform. If $I \subseteq \mathbb{R}$ is an interval, then $S_{I}$ is the Riesz projection adjusted to the interval $I$, i.e.,

$$
S_{I} f(t)=\int_{I} \hat{f}(s) e^{2 \pi \mathrm{i} s t} d s .
$$

The following remarkable inequality was proved by J. L. Rubio de Francia in [9]. For every $2 \leq p<\infty$, there is a constant $c_{p}$ such that for every collection of pairwise disjoint intervals $\left(I_{j}\right)_{j=1}^{\infty}$, the following estimate holds:

$$
\left\|\left(\sum_{j=1}^{\infty}\left|S_{I_{j}} f\right|^{2}\right)^{\frac{1}{2}}\right\|_{L^{p}(\mathbb{R})} \leq c_{p}\|f\|_{L^{p}(\mathbb{R})}, \quad \forall f \in L^{p}(\mathbb{R}) .
$$

In this note, we shall discuss a version of the theorem above when functions take values in a Banach space $X$. Let $\left(\varepsilon_{k}\right)_{k>1}$ be the system of Rademacher functions on $[0,1]$. The $\operatorname{space} \operatorname{Rad}(X)$ is the closure in $L^{p}([0,1] ; X), 1 \leq p<\infty$, of all $X$-valued functions of the form

$$
g(\omega)=\sum_{k=1}^{n} \varepsilon_{k}(\omega) x_{k}, \quad x_{k} \in X, \quad n \geq 1 .
$$

Mathematics Subject Classification (2010): Primary 46B20; Secondary 46B42.

Keywords: Littlewood-Paley-Rubio de Francia inequality, UMD space of type 2, Banach lattices. 
The above definition is independent of $1 \leq p<\infty$. It follows from the Khintchine-Kahane inequality (see [6]). In fact, the above fact is a consequence of the so-called contraction principle. It states that, for every sequence of elements $\left\{x_{j}\right\}_{j=1}^{\infty} \subseteq X$ and sequence of complex numbers $\left\{\alpha_{j}\right\}_{j=1}^{\infty}$ such that $\left|\alpha_{j}\right| \leq 1$ for $j \geq 1$, the following inequality holds:

$$
\left\|\sum_{j=1}^{\infty} \alpha_{j} \epsilon_{j} x_{j}\right\|_{L^{p}(\mathbb{R}, \operatorname{Rad}(X))} \leq c_{p}\left\|\sum_{j=1}^{\infty} \epsilon_{j} x_{j}\right\|_{L^{p}(\mathbb{R}, \operatorname{Rad}(X))} .
$$

We shall employ this principle on numerous occasions in this paper.

Following [1], we shall call $X$ a space with the $\mathrm{LPR}_{p}$ property with $2 \leq p<\infty$, if there exists a constant $c>0$ such that for any collection of pairwise disjoint intervals $\left\{I_{j}\right\}_{j=1}^{\infty}$ we have that

$$
\left\|\sum_{j=1}^{\infty} \varepsilon_{j} S_{I_{j}} f\right\|_{L^{p}(\mathbb{R} ; \operatorname{Rad}(X))} \leq c\|f\|_{L^{p}(\mathbb{R} ; X)}, \quad \forall f \in L^{p}(\mathbb{R} ; X) .
$$

It was proved in [5] that every space with the $\mathrm{LPR}_{p}$ property is necessarily UMD and of type 2 . It is an open problem whether the converse is true. It is also unknown whether $\mathrm{LPR}_{p}$ is independent of $p$. Note that Rubio de Francia's inequality says that $\mathbb{C}$ has the $\mathrm{LPR}_{p}$ property for every $2 \leq p<\infty$. By the Khintchine inequality and the Fubini theorem we see that any $L^{p}$-space with $2 \leq p<\infty$ has the $\mathrm{LPR}_{p}$ property. Using interpolation, we deduce that a Lorentz space $L^{p, r}$ has the $\mathrm{LPR}_{q}$ property for some indices $p, r$ and $q$. However, until recently there were no nontrivial examples of spaces with $\mathrm{LPR}_{p}$ found.

If $X$ is a Banach lattice, the estimate (1.2) admits a pleasant form, as in the scalar case:

$$
\left\|\left(\sum_{j=1}^{\infty}\left|S_{I_{j}} f\right|^{2}\right)^{\frac{1}{2}}\right\|_{L^{p}(\mathbb{R} ; X)} \leq c\|f\|_{L^{p}(\mathbb{R} ; X)}, \quad \forall f \in L^{p}(\mathbb{R} ; X) .
$$

We shall show that if the 2-concavification $X_{(2)}$ of $X$ is a UMD Banach lattice, then (1.3) holds for all $2<p<\infty$, so $X$ is a space with the $\mathrm{LPR}_{p}$ property. Recall that $X_{(2)}$ is the lattice defined by the following quasi-norm

$$
\|f\|_{X_{(2)}}=\left\||f|^{\frac{1}{2}}\right\|_{X}^{2}
$$

The space $X_{(2)}$ is a Banach lattice if and only if $X$ is 2-convex, i.e.,

$$
\left\|\left(\sum_{j=1}^{n}\left|f_{j}\right|^{2}\right)^{\frac{1}{2}}\right\|_{X} \leq\left(\sum_{j=1}^{n}\left\|f_{j}\right\|_{X}^{2}\right)^{\frac{1}{2}}
$$

We refer to [6] for more information on Banach lattices.

We shall also show that if $X$ is a Banach space (not necessarily a lattice) with the $\mathrm{LPR}_{q}$ property for some $q$, then $X$ has the $\mathrm{LPR}_{p}$ property for every $p \geq q$. 


\section{Dyadic decomposition}

For every interval $I \subseteq \mathbb{R}$, let $2 I$ be the interval of double length and the same centre as $I$. Let $\mathcal{I}=\left(I_{j}\right)_{j=1}^{\infty}$ be a collection of pairwise disjoint intervals. We set $2 \mathcal{I}=\left(2 I_{j}\right)_{j=1}^{\infty}$. The collection $\mathcal{I}$ is called well-distributed if there is a number $d$ such that each element of $2 \mathcal{I}$ intersects at most $d$ other elements of $2 \mathcal{I}$.

In this section, we fix a pairwise disjoint collection of intervals $\left(I_{j}\right)_{j=1}^{\infty}$ and we break each interval $I_{j}, j \geq 1$, into a number of smaller dyadic subintervals such that the new collection is well-distributed. This construction was employed in a number of earlier papers.

We start with two elementary remarks on estimate (1.2) or (1.3). Firstly, it suffices to consider a finite sequence $\left(I_{j}\right)_{j}$ of disjoint finite intervals. Secondly, by dilation, we may assume $\left|I_{j}\right| \geq 4$ for all $j$. Thus all sums on $j$ and $k$ in what follows are finite. Fix $j \geq 1$. Let $I_{j}=\left(a_{j}, b_{j}\right]$. Let $n_{j}=\max \left\{n \in \mathbb{N}: 2^{n+1} \leq b_{j}-a_{j}+4\right\}$. We first split $I_{j}$ into two subintervals with equal lengths:

$$
I_{j}^{a}=\left(a_{j}, \frac{a_{j}+b_{j}}{2}\right] \text { and } I_{j}^{b}=\left(\frac{a_{j}+b_{j}}{2}, b_{j}\right] .
$$

Then we decompose $I_{j}^{a}$ and $I_{j}^{b}$ into relative dyadic subintervals as follows:

$$
I_{j}^{a}=\bigcup_{k=1}^{n_{j}}\left(a_{j, k}, a_{j, k+1}\right] \text { and } I_{j}^{b}=\bigcup_{k=1}^{n_{j}}\left(b_{j, k+1}, b_{j, k}\right]
$$

where

$$
\begin{aligned}
& a_{j, k}=a_{j}-2+2^{k}, \quad 1 \leq k \leq n_{j}, \quad \text { and } \quad a_{j, n_{j}+1}=\frac{a_{j}+b_{j}}{2} \\
& b_{j, k}=b_{j}+2-2^{k}, \quad 1 \leq k \leq n_{j}, \quad \text { and } \quad b_{j, n_{j}+1}=\frac{a_{j}+b_{j}}{2}
\end{aligned}
$$

Let

$$
I_{j, k}^{a}=\left(a_{j, k}, a_{j, k+1}\right], \quad I_{j, k}^{b}=\left(b_{j, k+1}, b_{j, k}\right]
$$

for $1 \leq k \leq n_{j}$ and let $I_{j, k}^{a}, I_{j, k}^{b}$ be the empty set for the other $k$ 's. Also put

$\tilde{I}_{j, n_{j}}^{a}=\left(a_{j}-2+2^{n_{j}}, a_{j}-2+2^{n_{j}+1}\right] \quad$ and $\quad \tilde{I}_{j, n_{j}}^{b}=\left(b_{j}+2-2^{n_{j}+1}, b_{j}+2-2^{n_{j}}\right]$.

Lemma 2.1. A Banach space $X$ has the $\mathrm{LPR}_{p}$ property if there is a constant $c>0$ such that

$$
\max _{u=a, b}\left\|\sum_{j=1}^{\infty} \varepsilon_{j} \sum_{k=1}^{n_{j}} \varepsilon_{k}^{\prime} S_{I_{j, k}^{u}} f\right\|_{L^{p}\left(\mathbb{R} ; \operatorname{Rad}_{2}(X)\right)} \leq c\|f\|_{L^{p}(\mathbb{R} ; X)}, \quad \forall f \in L^{p}(\mathbb{R} ; X),
$$

where $\operatorname{Rad}_{2}(X)=\operatorname{Rad}\left(\operatorname{Rad}^{\prime}(X)\right)$ and $\operatorname{Rad}^{\prime}(X)$ is the space with respect to another copy of the Rademacher system $\left(\varepsilon_{k}^{\prime}\right)_{k \geq 1}$. 
Observe that if (2.1) holds for every family of intervals $\left(I_{j}\right)_{j=1}^{\infty}$, then $X$ is a UMD space. Indeed, (2.1) implies that

$$
\left\|S_{I_{j, k}^{u}} f\right\|_{L^{p}(\mathbb{R}, X)} \leq c\|f\|_{L^{p}(\mathbb{R}, X)}, \quad u=a, b, j \geq 1,1 \leq k \leq n_{j} .
$$

That is, by adjusting the choice of intervals, it implies that every projection $S_{I}$ is bounded on $L^{p}(\mathbb{R}, X)$ and

$$
\sup _{I \subseteq \mathbb{R}}\left\|S_{I}\right\|_{L^{p}(\mathbb{R}, X) \mapsto L^{p}(\mathbb{R}, X)}<+\infty .
$$

The latter is equivalent to the fact that $X$ is UMD (see [3]).

Proof. Let $f \in L^{p}(\mathbb{R} ; X)$. Then

$$
\left\|\sum_{j=1}^{\infty} \varepsilon_{j} S_{I_{j}} f\right\|_{L^{p}(\mathbb{R} ; \operatorname{Rad}(X))} \leq\left\|\sum_{j=1}^{\infty} \varepsilon_{j} S_{I_{j}^{a}} f\right\|_{L^{p}(\mathbb{R} ; \operatorname{Rad}(X))}+\left\|\sum_{j=1}^{\infty} \varepsilon_{j} S_{I_{j}^{b}} f\right\|_{L^{p}(\mathbb{R} ; \operatorname{Rad}(X))} .
$$

Using the subintervals $I_{j, k}^{a}$ and the contraction principle, we write

$$
\begin{aligned}
\left\|\sum_{j=1}^{\infty} \varepsilon_{j} S_{I_{j}^{a}} f\right\|_{L^{p}(\mathbb{R} ; \operatorname{Rad}(X))} & =\left\|\sum_{j=1}^{\infty} \sum_{k=1}^{n_{j}} \varepsilon_{j} S_{I_{j, k}^{a}} f\right\|_{L^{p}(\mathbb{R} ; \operatorname{Rad}(X))} \\
& \sim\left\|\sum_{j=1}^{\infty} \sum_{k=1}^{n_{j}} \varepsilon_{j} \exp \left(-2 \pi \mathrm{i} a_{j} \cdot\right) S_{I_{j, k}^{a}} f\right\|_{L^{p}(\mathbb{R} ; \operatorname{Rad}(X))}
\end{aligned}
$$

Note that

$$
\exp \left(-2 \pi \mathrm{i} a_{j} \cdot\right) S_{I_{j, k}^{a}} f=S_{I_{j, k}^{a}-a_{j}}\left[\exp \left(-2 \pi \mathrm{i} a_{j} \cdot\right) f\right]
$$

and

$$
I_{j, k}^{a}-a_{j}=\left(2^{k}-2,2^{k+1}-2\right], k<n_{j} ; \quad I_{j, n_{j}}^{a}-a_{j} \subseteq\left(2^{n_{j}}-2,2^{n_{j}+1}-2\right] .
$$

Recall that $X$ is a UMD space. Therefore, applying Bourgain's Fourier multiplier theorem (see [3]) to the function

$$
\left.\sum_{j=1}^{\infty} \sum_{k=1}^{n_{j}} \varepsilon_{j} \exp \left(-2 \pi \mathrm{i} a_{j} \cdot\right) S_{I_{j, k}^{a}} f \in L^{p}(\mathbb{R} ; \operatorname{Rad}(X))\right),
$$

we obtain (the contraction principle being used in the last step)

$$
\begin{aligned}
\| \sum_{j=1}^{\infty} \sum_{k=1}^{n_{j}} & \varepsilon_{j} \exp \left(-2 \pi \mathrm{i} a_{j} \cdot\right) S_{I_{j, k}^{a}} f \|_{L^{p}(\mathbb{R} ; \operatorname{Rad}(X))} \\
& \sim\left\|\sum_{j=1}^{\infty} \sum_{k=1}^{n_{j}} \varepsilon_{j} \varepsilon_{k}^{\prime} \exp \left(-2 \pi \mathrm{i} a_{j} \cdot\right) S_{I_{j, k}^{a}} f\right\|_{L^{p}\left(\mathbb{R} ; \operatorname{Rad}_{2}(X)\right)} \\
& \sim\left\|\sum_{j=1}^{\infty} \sum_{k=1}^{n_{j}} \varepsilon_{j} \varepsilon_{k}^{\prime} S_{I_{j, k}^{a}} f\right\|_{L^{p}\left(\mathbb{R} ; \operatorname{Rad}_{2}(X)\right)} .
\end{aligned}
$$


Similarly,

$$
\left\|\sum_{j=1}^{\infty} \varepsilon_{j} S_{I_{j}^{b}} f\right\|_{L^{p}(\mathbb{R} ; \operatorname{Rad} X)} \sim\left\|\sum_{j=1}^{\infty} \sum_{k=1}^{n_{j}} \varepsilon_{j} \varepsilon_{k}^{\prime} S_{I_{j, k}^{b}} f\right\|_{L^{p}\left(\mathbb{R} ; \operatorname{Rad}_{2}(X)\right)} .
$$

Combining the preceding estimates, we get

$$
\begin{aligned}
& \left\|\sum_{j=1}^{\infty} \varepsilon_{j} S_{I_{j}} f\right\|_{L^{p}(\mathbb{R} ; \operatorname{Rad} X)} \\
& \quad \leq c_{p}\left[\left\|\sum_{j=1}^{\infty} \sum_{k=1}^{n_{j}} \varepsilon_{j} \varepsilon_{k}^{\prime} S_{I_{j, k}^{a}} f\right\|_{L^{p}\left(\mathbb{R} ; \operatorname{Rad}_{2}(X)\right)}+\left\|\sum_{j=1}^{\infty} \sum_{k=1}^{n_{j}} \varepsilon_{j} \varepsilon_{k}^{\prime} S_{I_{j, k}^{b}} f\right\|_{L^{p}\left(\mathbb{R} ; \operatorname{Rad}_{2}(X)\right)}\right] .
\end{aligned}
$$

Let us observe that, if $X$ is a UMD space, the argument in the proof above shows that

$$
\left\|\sum_{j=1}^{\infty} \varepsilon_{j} S_{I_{j}} f\right\|_{L^{p}(\mathbb{R} ; \operatorname{Rad} X)} \lesssim \max _{u=a, b}\left\|\sum_{j=1}^{\infty} \varepsilon_{j} \sum_{k=1}^{n_{j}} \varepsilon_{k}^{\prime} S_{I_{j, k}^{u}} f\right\|_{L^{p}\left(\mathbb{R} ; \operatorname{Rad}_{2}(X)\right)} .
$$

Moreover, the argument can be reversed to show the opposite estimate (see the proof of (4.1) below). This observation is summarised in the following remark.

Remark 2.2. $\quad$ i) If $X$ is a UMD space, then

$$
\left\|\sum_{j=1}^{\infty} \varepsilon_{j} S_{I_{j}} f\right\|_{L^{p}(\mathbb{R} ; \operatorname{Rad} X)} \lesssim \max _{u=a, b}\left\|\sum_{j=1}^{\infty} \varepsilon_{j} \sum_{k=1}^{n_{j}} \varepsilon_{k}^{\prime} S_{I_{j, k}^{u}} f\right\|_{L^{p}\left(\mathbb{R} ; \operatorname{Rad}_{2}(X)\right)} .
$$

ii) If $\mathcal{I}=\left(I_{j}\right)_{j \geq 1}$ is a collection of pairwise disjoint intervals and, for $u=a, b$, $\mathcal{I}_{u}=\left(I_{j, k}^{u}\right)_{j \geq 1,1 \leq k \leq n_{j}}$, then both collections $\mathcal{I}_{a}$ and $\mathcal{I}_{b}$ are well-distributed.

iii) If $X$ is a Banach lattice then it has the $\alpha$-property (see [7]). That is,

$$
\left\|\sum_{j, k=1}^{\infty} \varepsilon_{j} \varepsilon_{k}^{\prime} x_{j k}\right\|_{\operatorname{Rad}_{2}(X)} \sim\left\|\sum_{j, k=1}^{\infty} \varepsilon_{j k} x_{j k}\right\|_{\operatorname{Rad}(X)},
$$

where $\left(\varepsilon_{j k}\right)$ is an independent family of Rademacher functions.

iv) The above two observations imply that if $X$ is a Banach lattice, then it has the $\mathrm{LPR}_{p}$ property if and only if estimate (1.2) holds for every well-distributed collection of intervals $\mathcal{I}$. 


\section{LPR-estimate for Banach lattices}

Theorem 3.1. If $X$ is a Banach lattice such that $X_{(2)}$ is a UMD Banach space, then $X$ has the $\mathrm{LPR}_{p}$ property for every $2<p<\infty$.

We shall need the following remark for the proof.

Remark 3.2. If $X$ is UMD and $1<p<\infty$, then the family $\left\{S_{I}\right\}_{I \subseteq \mathcal{I}}$ is $R$-bounded (see [4]), i.e.,

$$
\left\|\sum_{I \subseteq \mathcal{I}} \epsilon_{I} S_{I} f_{I}\right\|_{L^{p}(\mathbb{R} ; \operatorname{Rad}(X))} \leq c_{X}\left\|\sum_{I \subseteq \mathcal{I}} \epsilon_{I} f_{I}\right\|_{L^{p}(\mathbb{R} ; \operatorname{Rad}(X))} .
$$

Proof of Theorem 3.1. The proof directly employs the pointwise estimate of [9]. We assume that $X$ is a Köthe function space on a measure space $(\Omega, \mu)$.

Let $f \in L_{\text {loc }}^{1}(\mathbb{R} ; X)$ and let $M(f)$ be the Hardy-Littlewood maximal function of $f$, i.e.,

$$
M(f)(t)=\sup _{\substack{I \subseteq \mathbb{R} \\ t \in I}} \frac{1}{|I|} \int_{I}|f(s)| d s
$$

and

$$
M_{2}(f)=\left[M|f|^{2}\right]^{\frac{1}{2}}
$$

Let

$$
f^{\sharp}(t)=\sup _{\substack{I \subseteq \mathbb{R} \\ t \in I}} \frac{1}{|I|} \int_{I}\left|f(s)-f_{I}\right| d s, \quad f_{I}=\frac{1}{|I|} \int_{I} f(s) d s .
$$

Note that $M(f)$ is a function of two variables $(t, \omega)$ : for each fixed $\omega, M(f)(\cdot, \omega)$ is the usual Hardy-Littlewood maximal function of $f(\cdot, \omega)$. The same remark applies to $M_{2}(f)$ and $f^{\sharp}$. For $f$ sufficiently nice (which will be assumed in the sequel), all these functions are well-defined.

Observe that due to Remark 2.2 we have only to show estimate (1.2) for a welldistributed family of intervals. Let us fix a family of pairwise disjoint intervals $\mathcal{I}$ and let us assume that $\mathcal{I}$ is well-distributed. Fix a Schwartz function $\psi(t)$ whose Fourier transform satisfies

$$
\chi_{[-1 / 2,1 / 2]} \leq \hat{\psi} \leq \chi_{[-1,1]} .
$$

If $I \in \mathcal{I}$, then we set

$$
\psi_{I}(t)=|I| \exp \left(2 \pi \mathrm{i} c_{I} t\right) \psi(|I| t),
$$

where $c_{I}$ is the centre of $I$. The Fourier transform of $\psi_{I}$ is adapted to $I$, i.e.,

$$
\chi_{I} \leq \hat{\psi}_{I} \leq \chi_{2 I}
$$

In particular,

$$
S_{I}(f)=\psi_{I} * S_{I}(f) .
$$


Consequently, from the Khintchine inequality and Remark 3.2,

$$
\left\|\left(\sum_{I \in \mathcal{I}}\left|S_{I}(f)\right|^{2}\right)^{\frac{1}{2}}\right\|_{L^{p}(\mathbb{R}, X)} \leq c_{p}\|G(f)\|_{L^{p}(\mathbb{R}, X)}, \quad 1<p<\infty,
$$

where

$$
G(f)=\left(\sum_{I \in \mathcal{I}}\left|\psi_{I} * f\right|^{2}\right)^{\frac{1}{2}}, \quad f \in L^{1}(\mathbb{R} ; X) .
$$

Thus, to finish the proof, we need to show that

$$
\|G(f)\|_{L^{p}(\mathbb{R}, X)} \leq c_{p}\|f\|_{L^{p}(\mathbb{R}, X)}, \quad 2<p<\infty .
$$

It was shown in $[9]$ that $G(f(\cdot, \omega))^{\sharp}$ is almost everywhere dominated by $M_{2}(f(\cdot, \omega))$, i.e.,

$$
G(f(\cdot, \omega))^{\sharp} \leq c M_{2}(f(\cdot, \omega)), \text { a.e. } \omega \in \Omega,
$$

for some universal $c>0$. Since

$$
G(f)(t, \omega)=G(f(\cdot, \omega))(t) \quad \text { and } \quad M_{2}(f)(t, \omega)=M_{2}(f(\cdot, \omega))(t), \quad t \in \mathbb{R}, \omega \in \Omega,
$$

we clearly have that

$$
G(f)^{\sharp} \leq c M_{2}(f) .
$$

Therefore,

$$
\left\|G(f)^{\sharp}\right\|_{L^{p}(\mathbb{R} ; X)} \leq c\left\|M_{2}(f)\right\|_{L^{p}(\mathbb{R} ; X)} .
$$

It remains to prove

$$
\|G(f)\|_{L^{p}(\mathbb{R} ; X)} \leq C\left\|G(f)^{\sharp}\right\|_{L^{p}(\mathbb{R} ; X)} \quad \text { and } \quad\left\|M_{2}(f)\right\|_{L^{p}(\mathbb{R} ; X)} \leq C\|f\|_{L^{p}(\mathbb{R} ; X)} .
$$

The second inequality above immediately follows from Bourgain's maximal inequality for UMD lattices (applied to $X_{(2)}$ here, see Theorem 3 in [10]):

$$
\left\|M_{2}(f)\right\|_{L^{p}(\mathbb{R} ; X)}^{2}=\left\|M\left(|f|^{2}\right)\right\|_{L^{\frac{p}{2}\left(\mathbb{R} ; X_{(2)}\right)}} \leq C\left\||f|^{2}\right\|_{L^{\frac{p}{2}\left(\mathbb{R} ; X_{(2)}\right)}}=C\|f\|_{L^{p}(\mathbb{R} ; X)}^{2} .
$$

It remains to show the first one. To this end we shall prove the following inequality (for a general $f$ instead of $G(f)$ ):

$$
\|f\|_{L^{p}(\mathbb{R} ; X)} \leq C\left\|f^{\sharp}\right\|_{L^{p}(\mathbb{R} ; X)} .
$$

This is again an immediate consequence of the following classical duality inequality (see page 146 of [12]):

$$
\left|\int_{\mathbb{R}} u v\right| \leq C \int_{\mathbb{R}} u^{\sharp} \mathcal{M}(v)
$$

for any $u \in L^{p}(\mathbb{R})$ and $v \in L^{p^{\prime}}(\mathbb{R})$, where $\mathcal{M}(v)$ denotes the grand maximal function of $v$. Note that $\mathcal{M}(v) \leq C M(v)$. Now let $g \in L^{p^{\prime}}\left(\mathbb{R} ; X^{*}\right)$ be a nice function. We then have

$$
\begin{aligned}
\left|\int_{\mathbb{R} \times \Omega} f g\right| & \leq C \int_{\mathbb{R} \times \Omega} f^{\sharp} M(g) \leq C\left\|f^{\sharp}\right\|_{L^{p}(\mathbb{R} ; X)}\|M(g)\|_{L^{p^{\prime}}\left(\mathbb{R} ; X^{*}\right)} \\
& \leq C\left\|f^{\sharp}\right\|_{L^{p}(\mathbb{R} ; X)}\|g\|_{L^{p^{\prime}\left(\mathbb{R} ; X^{*}\right)}},
\end{aligned}
$$


where we have used again Bourgain's maximal inequality for $g$ (noting that $X^{*}$ is also a UMD lattice). Therefore, taking the supremum over all $g$ in the unit ball of $L^{p^{\prime}}\left(\mathbb{R} ; X^{*}\right)$, we deduce the desired inequality, so prove the theorem.

Finally, observe that the proof above operates with individual functions. This, coupled with the UMD property of $X$, implies that $X$ can always be assumed separable and it can always be equipped with a weak unit.

\section{LPR property for general Banach spaces}

Let $X$ be a Banach space (not necessarily a lattice). We shall prove the following theorem:

Theorem 4.1. If $X$ has the $\mathrm{LPR}_{q}$ property for some $2 \leq q<\infty$, then $X$ has the $\mathrm{LPR}_{p}$ property for any $q \leq p<\infty$.

The proof of the theorem requires some lemmas.

Lemma 4.2. Assume that $X$ has the $\mathrm{LPR}_{q}$ property. Let $\left(I_{j}\right)_{j \geq 1}$ be a finite sequence of mutually disjoint intervals of $\mathbb{R}$ and $\left(I_{j, k}\right)_{k=1}^{n_{j}}$ be a finite family of $m u$ tually disjoint subintervals of $I_{j}$ for each $j \geq 1$. Assume that the relative position of $I_{j, k}$ in $I_{j}$ is independent of $j$, i.e., $I_{j, k}-a_{j}=I_{j^{\prime}, k}-a_{j}^{\prime}$ whenever both $I_{j, k}$ and $I_{j^{\prime}, k}$ are present (i.e., $\left.k \leq \min \left\{n_{j}, n_{j^{\prime}}\right\}\right)$, where $a_{j}$ is the left endpoint of $I_{j}$. Then

$$
\left\|\sum_{j=1}^{\infty} \sum_{k=1}^{n_{j}} \varepsilon_{j} \varepsilon_{k}^{\prime} S_{I_{j, k}} f\right\|_{L^{q}\left(\mathbb{R} ; \operatorname{Rad}_{2}(X)\right)} \leq c\|f\|_{L^{q}(\mathbb{R} ; X)}, \quad \forall f \in L^{q}(\mathbb{R} ; X) .
$$

Proof. We first assume that $\bigcup_{k=1}^{n_{j}} I_{j, k}=I_{j}$ for each $j \geq 1$. Note that

$$
S_{I_{j, k}} f=\exp \left(2 \pi \mathrm{i} a_{j} \cdot\right) S_{I_{j, k}-a_{j}}\left(\exp \left(-2 \pi \mathrm{i} a_{j} \cdot\right) f\right) .
$$

Thus, by the contraction principle,

$$
\left\|\sum_{j=1}^{\infty} \sum_{k=1}^{n_{j}} \varepsilon_{j} \varepsilon_{k}^{\prime} S_{I_{j, k}} f\right\|_{q} \sim\left\|\sum_{k=1}^{\infty} \varepsilon_{k}^{\prime} \sum_{j: n_{j} \geq k} \varepsilon_{j} S_{I_{j, k}-a_{j}}\left(\exp \left(-2 \pi \mathrm{i} a_{j} \cdot\right) f\right)\right\|_{q} .
$$

Since $X$ has the $\mathrm{LPR}_{q}$ property, so does $\operatorname{Rad}(X)$. Let us apply this property of $\operatorname{Rad}(X)$ to the intervals $\left(\tilde{I}_{k}\right)_{k \geq 1}$ where $\tilde{I}_{k}=I_{j, k}-a_{j}$, for some $j$ such that $n_{j} \geq k$ (for any such $j$ the interval $I_{j, k}-a_{j}$ is independent of $j$ by the assumptions of the lemma). We apply this property to the function

$$
\sum_{k=1}^{\infty} \sum_{j: n_{j} \geq k} \varepsilon_{j} S_{I_{j, k}-a_{j}}\left(\exp \left(-2 \pi \mathrm{i} a_{j} \cdot\right) f\right)=\sum_{k=1}^{\infty} S_{\tilde{I}_{k}}\left[\sum_{j: n_{j} \geq k} \epsilon_{j}\left(\exp \left(-2 \pi \mathrm{i} a_{j} \cdot\right) f\right)\right] .
$$


We obtain

$$
\begin{aligned}
& \| \sum_{k=1}^{\infty} \varepsilon_{k}^{\prime} \sum_{j: n_{j} \geq k} \varepsilon_{j} S_{I_{j, k}-a_{j}}\left(\exp \left(-2 \pi \mathrm{i} a_{j} \cdot\right) f\right) \|_{q} \\
& \leq c\left\|\sum_{k=1}^{\infty} \sum_{j: n_{j} \geq k} \varepsilon_{j} S_{I_{j, k}-a_{j}}\left(\exp \left(-2 \pi \mathrm{i} a_{j} \cdot\right) f\right)\right\|_{q} \\
& \sim c\left\|\sum_{j=1}^{\infty} \sum_{k=1}^{n_{j}} \varepsilon_{j} S_{I_{j, k}} f\right\|_{q}=c\left\|\sum_{j=1}^{\infty} \varepsilon_{j} S_{I_{j}} f\right\|_{q} \leq c\|f\|_{q} .
\end{aligned}
$$

Assume now that $\bigcup_{k=1}^{n_{j}} I_{j, k} \neq I_{j}$ for some $j$. In this case, consider the family of intervals $\left(\tilde{I}_{k}\right)_{k=1}^{\infty}$ introduced above. Observe that every $\tilde{I}_{k} \subseteq[0,+\infty)$. Observe also that the the right ends of the intervals $\left(I_{j}-a_{j}\right)_{j \geq 1}$, that is the points $b_{j}-a_{j}$, do not belong to the union $\cup_{k=1}^{\infty} \tilde{I}_{k}$. Let $\left(\tilde{I}_{\ell}\right)_{\ell=1}^{\infty}$ be the family of disjoint intervals such that

$$
\bigcup_{\ell=1}^{\infty} \tilde{I}_{\ell}=[0,+\infty) \backslash \bigcup_{k=1}^{\infty} \tilde{I}_{k}
$$

and such that neither of the points $\left(b_{j}-a_{j}\right)_{j=1}^{\infty}$ is inner for some $\tilde{I}_{\ell}$. Let also $m_{j}$ be the maximum number such that the intervals $\tilde{I}_{\ell}$ with $\ell \leq m_{j}$ are all to the left of the point $b_{j}-a_{j}$. Set $I_{j, \ell}=\tilde{I}_{\ell}+a_{j}$. Then,

$$
I_{j}=\bigcup_{k=1}^{n_{j}} I_{j, k}+\bigcup_{\ell=1}^{m_{j}} I_{j, \ell} .
$$

It is clear that the relative position of $\left(I_{j, k}\right)_{k=1}^{n_{j}} \cup\left(I_{j, \ell}\right)_{\ell=1}^{m_{j}}$ in $I_{j}$ is again independent of $j$.

Before we proceed, let us re-index the intervals $\left(I_{j, k}\right)_{k=1}^{n_{j}}$ and $\left(I_{j, \ell}\right)_{\ell=1}^{m_{j}}$ into a family $\left(I_{j, s}\right)_{s=1}^{m_{j}+n_{j}}$ as follows. We arrange these intervals from left to right within $I_{j}$ and index them sequentially from 1 up to $n_{j}+m_{j}$. Moreover, let $K_{j} \subseteq\left[1, n_{j}+m_{j}\right]$ be the subset corresponding to the first family of intervals and $L_{j} \subseteq\left[1, n_{j}+m_{j}\right]$ be the subset of indices corresponding to the second family of intervals. Observe that, if $K=\cup_{j=1}^{\infty} K_{j}$ and $L=\cup_{j=1}^{\infty} L_{j}$, then, for every $j, K_{j}=K \cap\left[1, n_{j}+m_{j}\right]$ and, similarly, $L_{j}=L \cap\left[1, n_{j}+m_{j}\right]$. Thus by the previous part we get

$$
\left\|\sum_{j=1}^{\infty} \sum_{s=1}^{n_{j}+m_{j}} \epsilon_{j} \epsilon_{s}^{\prime} S_{I_{j, s}} f\right\|_{q} \leq c_{q}\|f\|_{q} .
$$

Observe also that

$$
\begin{aligned}
\sum_{j=1}^{\infty} \sum_{s=1}^{n_{j}+m_{j}} \epsilon_{j} \epsilon_{s}^{\prime} S_{I_{j, s}} f & =\sum_{s=1}^{\infty} \sum_{j: n_{j}+m_{j} \geq s} \epsilon_{j} \epsilon_{s}^{\prime} S_{I_{j, s}} f \\
& =\sum_{s \in K} \sum_{j: n_{j}+m_{j} \geq s} \epsilon_{j} \epsilon_{s}^{\prime} S_{I_{j, s}} f+\sum_{s \in L} \sum_{j: n_{j}+m_{j} \geq s} \epsilon_{j} \epsilon_{s}^{\prime} S_{I_{j, s}} f .
\end{aligned}
$$


Thus, by taking the projection onto the subspace spanned by $\left\{\epsilon_{s}^{\prime}\right\}_{s \in K}$, we obtain

$$
\left\|\sum_{s \in K} \sum_{j: n_{j}+m_{j} \geq s} \epsilon_{j} \epsilon_{s}^{\prime} S_{I_{j, s}} f\right\|_{q} \leq c_{q}\|f\|_{q} .
$$

Finally, we observe that

$$
\sum_{s \in K} \sum_{j: n_{j}+m_{j} \geq s} \epsilon_{j} \epsilon_{s}^{\prime} S_{I_{j, s}} f=\sum_{j=1}^{\infty} \sum_{k=1}^{n_{j}} \epsilon_{j} \epsilon_{k}^{\prime} S_{I_{j, k}} f .
$$

Hence the lemma is proved.

Next lemma is interesting in its own right. We shall only need its first part.

Lemma 4.3. Let $Y$ be a Banach space. Let $(\Sigma, \nu)$ be a measure space and $\left(h_{j}\right) \subset$ $L^{2}(\Sigma)$ a finite sequence.

i) If $Y$ is of cotype 2 and there exists a constant $c$ such that

$$
\left\|\sum_{j} \alpha_{j} h_{j}\right\|_{2} \leq c\left(\sum_{j}\left|\alpha_{j}\right|^{2}\right)^{1 / 2}, \quad \forall \alpha_{j} \in \mathbb{C},
$$

then

$$
\left\|\sum_{j} h_{j} a_{j}\right\|_{L^{2}(\Sigma ; Y)} \leq c^{\prime}\left\|\sum \varepsilon_{j} a_{j}\right\|_{\operatorname{Rad}(Y)}, \quad \forall a_{j} \in Y .
$$

ii) If $Y$ is of type 2 and there exists a constant $c$ such that

$$
\left(\sum_{j}\left|\alpha_{j}\right|^{2}\right)^{1 / 2} \leq c\left\|\sum_{j} \alpha_{j} h_{j}\right\|_{2}, \quad \forall \alpha_{j} \in \mathbb{C},
$$

then

$$
\left\|\sum \varepsilon_{j} a_{j}\right\|_{\operatorname{Rad}(Y)} \leq c^{\prime}\left\|\sum_{j} h_{j} a_{j}\right\|_{L^{2}(\Sigma ; Y)}, \quad \forall a_{j} \in Y .
$$

Proof. i) Let $\left(a_{j}\right) \subset Y$ be a finite sequence. Consider the operator $u: \ell^{2} \rightarrow Y$ defined by

$$
u(\alpha)=\sum_{j} \alpha_{j} a_{j}, \quad \forall \alpha=\left(\alpha_{j}\right) \in \ell^{2} .
$$

It is well known (see Lemma 3.8 and Theorem 3.9 in [8]) that

$$
\pi_{2}(u) \leq c_{0}\left\|\sum \varepsilon_{j} a_{j}\right\|_{\operatorname{Rad}(Y)},
$$

where $c_{0}$ is a constant depending only on the cotype 2 constant of $Y$. Let $h(\sigma)=$ $\left(h_{j}(\sigma)\right)_{j}$ for $\sigma \in \Sigma$. Then by the assumption on $\left(h_{j}\right)$ we get

$$
\begin{aligned}
\left\|\sum_{j} h_{j} a_{j}\right\|_{L^{2}(\Sigma ; Y)} & =\pi_{2}(u) \sup \left\{\left(\int_{\Sigma}\left|\sum_{j} \xi_{j} h_{j}(s)\right|^{2} d s\right)^{1 / 2}: \xi \in \ell^{2},\|\xi\|_{2} \leq 1\right\} \\
& \leq c^{\prime}\left\|\sum \varepsilon_{j} a_{j}\right\|_{\operatorname{Rad}(Y)} .
\end{aligned}
$$


ii) Let $H$ be the linear span of $\left(h_{j}\right)$ in $L^{2}(\Sigma)$. Let $h_{j}^{*}$ be the functional on $H$ such that $h_{j}^{*}\left(h_{k}\right)=\delta_{j, k}$. We extend $h_{j}^{*}$ to all of $L^{2}(\Sigma)$ by setting $h_{j}^{*}=0$ on $H^{\perp}$. Then $h_{j}^{*} \in L^{2}(\Sigma)$ and the assumption implies that

$$
\left\|\sum_{j} \beta_{j} h_{j}^{*}\right\|_{2} \leq c\left(\sum_{j}\left|\beta_{j}\right|^{2}\right)^{1 / 2}, \quad \forall \beta_{j} \in \mathbb{C} .
$$

Now let $\left(a_{j}^{*}\right) \subset Y^{*}$ be a finite sequence. Applying i) to $Y^{*}$ and $\left(h_{j}^{*}\right)$ we obtain

$$
\begin{aligned}
\left|\sum_{j}\left\langle a_{j}^{*}, a_{j}\right\rangle\right| & =\left|\left\langle\sum_{j} h_{j}^{*} a_{j}^{*}, \sum_{j} h_{j} a_{j}\right\rangle\right| \leq\left\|\sum_{j} h_{j}^{*} a_{j}^{*}\right\|_{L^{2}\left(\Sigma ; Y^{*}\right)}\left\|\sum_{j} h_{j} a_{j}\right\|_{L^{2}(\Sigma ; Y)} \\
& \leq c^{\prime}\left\|\sum_{j} \varepsilon_{j} a_{j}^{*}\right\|_{\operatorname{Rad}\left(Y^{*}\right)}\left\|\sum_{j} h_{j} a_{j}\right\|_{L^{2}(\Sigma ; Y)} .
\end{aligned}
$$

Taking the supremum over $\left(a_{j}^{*}\right) \subset Y^{*}$ such that $\left\|\sum \varepsilon_{j} a_{j}^{*}\right\|_{\operatorname{Rad}\left(Y^{*}\right)} \leq 1$, we get the assertion.

Now we proceed to the proof of Theorem 4.1. It is divided into several steps.

The singular integral operator $\boldsymbol{T}$. Let $\left(I_{j}\right)_{j}$ be a family of disjoint finite intervals and $\psi$ be a Schwartz function as in Sections 2 and 3. We keep the notation introduced there. We now set up an appropriate singular integral operator corresponding to (2.1). It suffices to consider the family $\left(I_{j, k}^{a}\right)_{j, k},\left(I_{j, k}^{b}\right)_{j, k}$ being treated similarly. Henceforth, we shall denote $I_{j, k}^{a}$ simply by $I_{j, k}$. Let $c_{j, k}=$ $a_{j, k}+2^{k-1}$ for $1 \leq k \leq n_{j}$. Note that $c_{j, k}$ is the centre of $I_{j, k}$ if $k<n_{j}$ and of $\tilde{I}_{j, k}$ if $k=n_{j}$. Define

$$
\psi_{j, k}(x)=2^{k} \exp \left(2 \pi \mathrm{i} c_{j, k} x\right) \psi\left(2^{k} x\right)
$$

so that the Fourier transform of $\psi_{j, k}$ is adapted to $I_{j, k}$, i.e.,

$$
\chi_{I_{j, k}} \leq \widehat{\psi}_{j, k} \leq \chi_{2 I_{j, k}} \text { for } k<n_{j} \quad \text { and } \quad \chi_{\tilde{I}_{j, n_{j}}} \leq \widehat{\psi}_{j, n_{j}} \leq \chi_{2 \tilde{I}_{j, n_{j}}} .
$$

We should emphasize that our choice of $c_{j, k}$ is different from that of Rubio de Francia in [9], which was $c_{j, k}=n_{j, k} 2^{k}$ for some integer $n_{j, k}$. Rubio de Francia's choice makes his calculations easier than ours in the scalar-valued case. The sole reason for our choice of $c_{j, k}$ is that $c_{j, k}$ splits into a sum of two terms depending on $j$ and $k$ separately. Namely, $c_{j, k}=a_{j}-2+2^{k}+2^{k-1}$. By (4.2),

$$
S_{I_{j, k}} f=S_{I_{j, k}} \psi_{j, k} * f .
$$

We then deduce, by the splitting property and Remark 3.2,

$$
\left\|\sum_{j, k} \varepsilon_{j} \varepsilon_{k}^{\prime} S_{I_{j, k}} f\right\|_{p} \leq c_{p}\left\|\sum_{j, k} \varepsilon_{j} \varepsilon_{k}^{\prime} \psi_{j, k} * f\right\|_{p} .
$$


Now write

$$
\begin{aligned}
\psi_{j, k} * f(x) & =\int 2^{k} \psi\left(2^{k}(x-y)\right) \exp \left(2 \pi \mathrm{i} c_{j, k}(x-y)\right) f(y) d y \\
& =\exp \left(2 \pi \mathrm{i} c_{j, k} x\right) \int 2^{k} \psi\left(2^{k}(x-y)\right) \exp \left(-2 \pi \mathrm{i} c_{j, k} y\right) f(y) d y \\
& =\exp \left(2 \pi \mathrm{i} c_{j, k} x\right) \int K_{j, k}(x, y) f(y) d y
\end{aligned}
$$

where

$$
K_{j, k}(x, y)=2^{k} \psi\left(2^{k}(x-y)\right) \exp \left(-2 \pi \mathrm{i} c_{j, k} y\right) .
$$

Using the splitting property of the $c_{j, k}$ mentioned previously and the contraction principle, for every $x \in \mathbb{R}$ we have

$$
\begin{aligned}
\left\|\sum_{j, k} \varepsilon_{j} \varepsilon_{k}^{\prime} \psi_{j, k} * f(x)\right\|_{\operatorname{Rad}_{2}(X)} & =\left\|\sum_{j, k} \varepsilon_{j} \varepsilon_{k}^{\prime} \exp \left(2 \pi \mathrm{i} c_{j, k} x\right) \int K_{j, k}(x, y) f(y) d y\right\|_{\operatorname{Rad}_{2}(X)} \\
& \sim\left\|\sum_{j, k} \varepsilon_{j} \varepsilon_{k}^{\prime} \int K_{j, k}(x, y) f(y) d y\right\|_{\operatorname{Rad}_{2}(X)} .
\end{aligned}
$$

Thus we are led to introduce the vector-valued kernel $K$ :

$$
K(x, y)=\sum_{j, k} \varepsilon_{j} \varepsilon_{k}^{\prime} K_{j, k}(x, y) \in L^{2}(\Omega), \quad x, y \in \mathbb{R} .
$$

$K$ is also viewed as a kernel taking values in $B\left(X, \operatorname{Rad}_{2}(X)\right)$ by multiplication. Let $T$ be the associated singular integral operator:

$$
T(f)(x)=\int K(x, y) f(y) d y, \quad f \in L^{p}(\mathbb{R} ; X) .
$$

By the discussion above, inequality (2.1) is reduced to the boundedness of $T$ from $L^{p}(\mathbb{R} ; X)$ to $L^{p}\left(\mathbb{R} ; \operatorname{Rad}_{2}(X)\right)$ :

$$
\|T(f)\|_{p} \leq c_{p}\|f\|_{p}, \quad \forall f \in L^{p}(\mathbb{R} ; X) .
$$

The $\boldsymbol{L}^{q}$ boundedness of $\boldsymbol{T}$. We have the following:

Lemma 4.4. $T$ is bounded from $L^{q}(\mathbb{R} ; X)$ to $L^{q}\left(\mathbb{R} ; \operatorname{Rad}_{2}(X)\right)$.

Proof. Let $f \in L^{q}(\mathbb{R} ; X)$. By the previous discussion we have

$$
\|T f\|_{q} \sim\left\|\sum_{j, k} \varepsilon_{j} \varepsilon_{k}^{\prime} \psi_{j, k} * f\right\|_{q} .
$$

By (4.2),

$$
\sum_{j, k} \varepsilon_{j} \varepsilon_{k}^{\prime} \psi_{j, k} * f=\sum_{j, k} \varepsilon_{j} \varepsilon_{k}^{\prime} \psi_{j, k} *\left(S_{2 I_{j, k}} f\right) .
$$


Note that for each $j$ the last interval $I_{j, n_{j}}$ above should be the dyadic interval $\widetilde{I}_{j, n_{j}}$. We claim that

$$
\left\|\sum_{j, k} \varepsilon_{j} \varepsilon_{k}^{\prime} \psi_{j, k} * g_{j, k}\right\|_{q} \leq c\left\|\sum_{j, k} \varepsilon_{j} \varepsilon_{k}^{\prime} g_{j, k}\right\|_{q}, \quad \forall g_{j, k} \in L^{q}(\mathbb{R} ; X) .
$$

Indeed, using the splitting property of the $c_{j, k}$ we have

$$
\left\|\sum_{j, k} \varepsilon_{j} \varepsilon_{k}^{\prime} \psi_{j, k} * g_{j, k}\right\|_{q} \sim\left\|\sum_{j, k} \varepsilon_{j} \varepsilon_{k}^{\prime} \widetilde{\psi}_{j, k} * \widetilde{g}_{j, k}\right\|_{q}
$$

where

$$
\widetilde{\psi}_{j, k}(x)=2^{k} \psi\left(2^{k} x\right) \quad \text { and } \quad \widetilde{g}_{j, k}(x)=\exp \left(-2 \pi \mathrm{i} c_{j, k} x\right) g_{j, k}(x) .
$$

For $x \in \mathbb{R}$ define the operator $N(x): \operatorname{Rad}_{2}(X) \rightarrow \operatorname{Rad}_{2}(X)$ by

$$
N(x)\left(\sum_{j, k} \varepsilon_{j} \varepsilon_{k}^{\prime} a_{j, k}\right)=\sum_{j, k} \varepsilon_{j} \varepsilon_{k}^{\prime} \widetilde{\psi}_{j, k}(x) a_{j, k} .
$$

It is obvious that $N: \mathbb{R} \rightarrow B\left(\operatorname{Rad}_{2}(X)\right)$ is a smooth function and

$$
\sum_{j, k} \varepsilon_{j} \varepsilon_{k}^{\prime} \widetilde{\psi}_{j, k} * \widetilde{g}_{j, k}=N * \widetilde{g} \quad \text { with } \quad \widetilde{g}=\sum_{j, k} \varepsilon_{j} \varepsilon_{k}^{\prime} \widetilde{g}_{j, k} .
$$

It is also easy to check that $N$ satisfies Theorem 3.4 in [11]. $\operatorname{Since~} \operatorname{Rad}_{2}(X)$ is a UMD space, it follows from [11] that the convolution operator with $N$ is bounded on $L^{q}\left(\mathbb{R} ; \operatorname{Rad}_{2}(X)\right)$. Thus,

$$
\left\|\sum_{j, k} \varepsilon_{j} \varepsilon_{k}^{\prime} \widetilde{\psi}_{j, k} * \widetilde{g}_{j, k}\right\|_{q} \leq c\left\|\sum_{j, k} \varepsilon_{j} \varepsilon_{k}^{\prime} \widetilde{g}_{j, k}\right\|_{q} .
$$

Using again the splitting property of the $c_{j, k}$ and going back to the $g_{j, k}$, we prove the claim. Consequently, we have

$$
\|T(f)\|_{q} \leq c\left\|\sum_{j, k} \varepsilon_{j} \varepsilon_{k}^{\prime} S_{2 I_{j, k}} f\right\|_{q} .
$$

We split the family $\left\{2 I_{j, k}\right\}$ into three subfamilies $\left\{2 I_{j, 3 k+\ell}\right\}$ of disjoint intervals with $\ell \in\{0,1,2\}$. Accordingly, we have

$$
\|T(f)\|_{q} \leq c \sum_{\ell=0}^{2}\left\|\sum_{j, k} \varepsilon_{j} \varepsilon_{k}^{\prime} S_{2 I_{j, 3 k+\ell}} f\right\|_{q} .
$$

Each subfamily $\left\{2 I_{j, 3 k+\ell}\right\}_{j, k}$ satisfies the condition of Lemma 4.2. Hence,

$$
\left\|\sum_{j, k} \varepsilon_{j} \varepsilon_{k}^{\prime} S_{2 I_{j, 3 k+\ell}} f\right\|_{q} \leq c\|f\|_{q} .
$$

Thus the lemma is proved. 
An estimate on the kernel $\boldsymbol{K}$. This subsection contains the key estimate on the kernel $K$ defined in (4.4). Fix $x, z \in \mathbb{R}$ and an integer $m \geq 1$. Let

$$
I_{m}(x, z)=\left\{y \in \mathbb{R}: 2^{m}|x-z|<|y-z| \leq 2^{m+1}|x-z|\right\} .
$$

Lemma 4.5. If $X^{*}$ is of cotype 2 and if $\left(\lambda_{j, k}\right) \subset X^{*}$, then

$$
\int_{I_{m}(x, z)}\left\|\sum_{j, k}\left[K_{j, k}(x, y)-K_{j, k}(z, y)\right] \lambda_{j, k}\right\|_{X^{*}}^{2} d y \leq c \frac{\left\|\sum_{j, k} \varepsilon_{j} \varepsilon_{k}^{\prime} \lambda_{j, k}\right\|_{\operatorname{Rad}_{2}\left(X^{*}\right)}^{2}}{2^{5 m / 3}|x-z|} .
$$

Proof. Let $\left(\lambda_{j, k}\right) \subset X^{*}$ such that

$$
\left\|\sum_{j, k} \varepsilon_{j} \varepsilon_{k}^{\prime} \lambda_{j, k}\right\|_{\operatorname{Rad}_{2}\left(X^{*}\right)} \leq 1 .
$$

By the definition of $K_{j, k}$ in (4.3), we have

$$
\sum_{j, k}\left[K_{j, k}(x, y)-K_{j, k}(z, y)\right] \lambda_{j, k}=\sum_{k} \mu_{k} 2^{k}\left[\psi\left(2^{k}(x-y)\right)-\psi\left(2^{k}(z-y)\right)\right] q_{k}(y)
$$

where

$$
\mu_{k}=\left\|\sum_{j} \varepsilon_{j} \lambda_{j, k}\right\|_{\operatorname{Rad}\left(X^{*}\right)} \quad \text { and } \quad q_{k}(y)=\mu_{k}^{-1} \sum_{j} \lambda_{j, k} \exp \left(-2 \pi \mathrm{i} c_{j, k} y\right) .
$$

Since $\operatorname{Rad}\left(X^{*}\right)$ is of cotype 2 ,

$$
\sum_{k} \mu_{k}^{2} \leq c\left\|\sum_{k} \varepsilon_{k}^{\prime} \sum_{j} \varepsilon_{j} \lambda_{j, k}\right\|_{\operatorname{Rad}\left(\operatorname{Rad}\left(X^{*}\right)\right)}^{2} \leq c .
$$

Thus,

$$
\begin{aligned}
\int_{I_{m}(x, z)} & \left\|\sum_{j, k}\left[K_{j, k}(x, y)-K_{j, k}(z, y)\right] \lambda_{j, k}\right\|_{X^{*}}^{2} d y \\
& \leq \sum_{k} 2^{2 k} \sup _{y \in I_{m}(x, z)}\left|\psi\left(2^{k}(x-y)\right)-\psi\left(2^{k}(z-y)\right)\right|^{2} \int_{I_{m}(x, z)}\left\|q_{k}(y)\right\|_{X^{*}}^{2} d y .
\end{aligned}
$$

Note that for fixed $k$

$$
\left|c_{j, k}-c_{j^{\prime}, k}\right| \geq 2^{k}, \quad \forall j \neq j^{\prime} .
$$

Now we appeal to the following classical inequality on Dirichlet series with small gaps. Let $\left(\gamma_{j}\right)$ be a finite sequence of real numbers such that

$$
\gamma_{j+1}-\gamma_{j} \geq 1, \quad \forall j \geq 1
$$

Then, by Theorem 9.9 in Chapter $\mathrm{V}$ of [13], for any interval $I \subset \mathbb{R}$ and any sequence $\left(\alpha_{j}\right) \subset \mathbb{C}$,

$$
\int_{I}\left|\sum_{j} \alpha_{j} \exp \left(2 \pi \mathrm{i} \gamma_{j} y\right)\right|^{2} d y \leq c \max (|I|, 1) \sum_{j}\left|\alpha_{j}\right|^{2}
$$


where $c$ is an absolute constant. Applying this to the function $q_{k}$, using Lemma 4.3 and (4.6), we find

$$
\begin{aligned}
\int_{I_{m}(x, z)}\left\|q_{k}\right\|_{X^{*}}^{2} d y & \leq c 2^{-k} \max \left(2^{k}\left|I_{m}(x, z)\right|, 1\right) \mu_{k}^{-2}\left\|\sum_{j} \varepsilon_{j} \lambda_{j, k}\right\|_{\operatorname{Rad}\left(X^{*}\right)}^{2} \\
& =c \max \left(2^{m}|x-z|, 2^{-k}\right) .
\end{aligned}
$$

Let

$$
\begin{aligned}
k_{0} & =\min \left\{k \in \mathbb{N}: 2^{-k} \leq 2^{m}|x-z|\right\} \\
\text { and } \quad & k_{1}=\min \left\{k \in \mathbb{N}: 2^{-k} \leq 2^{2 m / 3}|x-z|\right\} .
\end{aligned}
$$

Note that $k_{0} \leq k_{1}$. For $k \leq k_{1}$ we have

$$
\left|\psi\left(2^{k}(x-y)\right)-\psi\left(2^{k}(z-y)\right)\right| \leq c 2^{k}|x-z| .
$$

Recall that $\psi$ is a Schwartz function, in particular $|x|^{2}|\psi(x)| \leq c$. Thus, for $k \geq k_{1}$, we have

$$
\left|\psi\left(2^{k}(x-y)\right)-\psi\left(2^{k}(z-y)\right)\right| \leq c 2^{-2 k}|y-z|^{-2} \leq c 2^{-2 k-2 m}|x-z|^{-2},
$$

where the second estimate comes from the fact that $y \in I_{m}(x, z)$. Let

$$
\alpha_{k}=2^{2 k} \sup _{y \in I_{m}(x, z)}\left|\psi\left(2^{k}(x-y)\right)-\psi\left(2^{k}(z-y)\right)\right|^{2} \int_{I_{m}(x, z)}\left\|q_{k}(y)\right\|_{X}^{2} d y
$$

Combining the preceding inequalities, we deduce the following estimates on $\alpha_{k}$ :

$$
\begin{aligned}
& \alpha_{k} \leq c 2^{2 k} 2^{2 k}|x-z|^{2} 2^{-k}=c 2^{3 k}|x-z|^{2} \text { for } k \leq k_{0} ; \\
& \alpha_{k} \leq c 2^{2 k} 2^{2 k}|x-z|^{2} 2^{m}|x-z|=c 2^{4 k} 2^{m}|x-z|^{3} \text { for } \quad k_{0}<k<k_{1} ; \\
& \alpha_{k} \leq c 2^{2 k}\left(2^{k+m}|x-z|\right)^{-4} 2^{m}|x-z|=c 2^{-2 k} 2^{-3 m}|x-z|^{-3} \text { for } k \geq k_{1} .
\end{aligned}
$$

Therefore,

$$
\begin{aligned}
\int_{I_{m}(x, z)} \| & \sum_{j, k}\left[K_{j, k}(x, y)-K_{j, k}(z, y)\right] \lambda_{j, k} \|_{X^{*}}^{2} d y \\
& \leq \sum_{1 \leq k \leq k_{0}} \alpha_{k}+\sum_{k_{0}<k<k_{1}} \alpha_{k}+\sum_{k \geq k_{1}} \alpha_{k} \\
& \leq c\left[2^{3 k_{0}}|x-z|^{2}+2^{4 k_{1}} 2^{m}|x-z|^{3}+2^{-2 k_{1}} 2^{-3 m}|x-z|^{-3}\right] \\
& \leq c 2^{-5 m / 3}|x-z|^{-1}
\end{aligned}
$$

This is the desired estimate for the kernel $K$. 
The $\boldsymbol{L}^{\infty}$-BMO boundedness. Recall that $T$ is the singular integral operator associated with the kernel $K$.

Lemma 4.6. The operator $T$ is bounded from $L^{\infty}(\mathbb{R} ; X)$ to $\operatorname{BMO}\left(\mathbb{R} ; \operatorname{Rad}_{2}(X)\right)$.

Proof. Recall that

$$
\|g\|_{\mathrm{BMO}(\mathbb{R} ; \mathrm{X})} \leq 2 \sup _{I \subseteq \mathbb{R}} \frac{1}{|I|} \int_{I}\left\|g(x)-b_{I}\right\|_{X} d x
$$

where $\left\{b_{I}\right\}_{I \subseteq \mathbb{R}} \subseteq X$ is any family of elements of $X$ assigned to each interval $I \subseteq \mathbb{R}$. Fix a function $f \in L^{\infty}(\mathbb{R} ; X)$ with $\|f\|_{\infty} \leq 1$ and an interval $I \subset \mathbb{R}$. Let $z$ be the centre of $I$ and let

$$
b_{I}=\int_{(2 I)^{c}} K(z, y) f(y) d y .
$$

Then, for $x \in I$,

$$
T f(x)-b_{I}=\int_{(2 I)^{c}}[K(x, y)-K(z, y)] f(y) d y+\int_{2 I} K(x, y) f(y) d y .
$$

Thus

$$
\begin{aligned}
\frac{1}{|I|} \int_{I} \| & T f(x)-b_{I} \|_{\operatorname{Rad}_{2}(X)} d x \\
\leq & \frac{1}{|I|} \int_{I}\left\|\int_{(2 I)^{c}}[K(x, y)-K(z, y)] f(y) d y\right\|_{\operatorname{Rad}_{2}(X)} d x \\
& \quad+\frac{1}{|I|} \int_{I}\left\|\int_{2 I} K(x, y) f(y) d y\right\|_{\operatorname{Rad}_{2}(X)} d x \\
& \stackrel{\text { def }}{=} A+B .
\end{aligned}
$$

By Lemma 4.4 we have

$$
B \leq|I|^{-1 / q}\left\|T\left(f \chi_{2 I}\right)\right\|_{q} \leq c .
$$

To estimate $A$, fix $x \in I$. Choose $\left(\lambda_{j, k}\right) \subset X^{*}$ such that

$$
\left\|\sum_{j, k} \varepsilon_{j} \varepsilon_{k}^{\prime} \lambda_{j, k}\right\|_{\operatorname{Rad}_{2}\left(X^{*}\right)} \leq 1
$$

and

$$
\begin{aligned}
\| \int_{(2 I)^{c}}[K(x, y)-K(z, y)] & f(y) d y \|_{\operatorname{Rad}_{2}(X)} \\
& \sim \sum_{j, k}\left\langle\lambda_{j, k}, \int_{(2 I)^{c}}\left[K_{j, k}(x, y)-K_{j, k}(z, y)\right] f(y) d y\right\rangle
\end{aligned}
$$


Then by Lemma 4.5, we find

$$
\begin{aligned}
\| \int_{(2 I)^{c}} & {[K(x, y)-K(z, y)] f(y) d y \|_{\operatorname{Rad}_{2}(X)} } \\
& \leq \int_{(2 I)^{c}}\left\|\sum_{j, k}\left[K_{j, k}(x, y)-K_{j, k}(z, y)\right] \lambda_{j, k}\right\|_{X^{*}} d y \\
& \leq \sum_{m=1}^{\infty}\left|I_{m}(x, z)\right|^{1 / 2}\left(\int_{I_{m}(x, z)}\left\|\sum_{j, k}\left[K_{j, k}(x, y)-K_{j, k}(z, y)\right] \lambda_{j, k}\right\|_{X^{*}}^{2} d y\right)^{1 / 2} \\
& \leq c \sum_{m=1}^{\infty}\left(2^{m}|x-z|\right)^{1 / 2}\left(2^{5 m / 3}|x-z|\right)^{-1 / 2} \leq \sum_{m=1}^{\infty} c 2^{-m / 3} \leq c .
\end{aligned}
$$

Therefore, $A \leq c$. Thus $T$ is bounded from $L^{\infty}(\mathbb{R} ; X)$ to $\operatorname{BMO}\left(\mathbb{R} ; \operatorname{Rad}_{2}(X)\right)$.

Combining the results of Lemma 4.6 and Lemma 4.4 and applying interpolation (see [2]), we immediately see that the operator $T$ is bounded from $L^{p}(\mathbb{R} ; X)$ to $L^{p}\left(\mathbb{R} ; \operatorname{Rad}_{2}(X)\right)$ for every $q<p<\infty$. Thus Theorem 4.1 is proved.

Remark 4.7. Let

$$
T(f)^{\sharp}(x)=\sup _{x \in I} \frac{1}{|I|} \int_{I}\left\|T(f)(y)-T(f)_{I}\right\|_{\operatorname{Rad}_{2}(X)} d y
$$

and

$$
M_{q}(f)(x)=\sup _{x \in I}\left(\frac{1}{|I|} \int_{I}\|f(y)\|_{X}^{q} d y\right)^{\frac{1}{q}} .
$$

Under the assumption of Theorem 4.1 one can show the following pointwise estimate:

$$
T(f)^{\sharp} \leq c M_{q}(f) .
$$

\section{References}

[1] Berkson, E., Gillespie, T. A. And Torrea, J. L.: Vector valued transference. In Functional space theory and its applications: Proceedings of International Conference \& 13th Academic Symposium in China (Wuhan, 2003), 1-27. Research Information Limited UK, 2004.

[2] Blasco, O. ANd Xu, Q. H.: Interpolation between vector-valued Hardy spaces. J. Funct. Anal. 102 (1991), 331-359.

[3] Bourgain, J.: Vector-valued singular integrals and the $H^{1}$-BMO duality. In Probability theory and harmonic analysis (Cleveland, Ohio, 1983), 1-19. Monogr. Textbooks Pure Appl. Math., 98. Dekker, New York, 1986.

[4] Clément, P., de Pagter, B., Sukochev, F. A. And Witvliet, H.: Schauder decomposition and multiplier theorems. Studia Math. 138 (2000), 135-163.

[5] Hytönen, T. P., Torrea, J. L. And Yakubovich, D. V.: The Littlewood-PaleyRubio de Francia property of a Banach space for the case of equal intervals. Proc. Roy. Soc. Edinburgh Sect. A 139 (2009), 819-832. 
[6] Lindenstrauss, J. And Tzafriri, L.: Classical Banach spaces. II. Function spaces. Results in Mathematics and Related Areas, 97. Springer, Berlin-New York, 1979.

[7] Pisier, G.: Some results on Banach spaces without local unconditional structures. Compositio Math. 37 (1978) 3-19.

[8] Pisier, G.: Factorization of linear operators and geometry of Banach spaces. CBMS Regional Conference Series in Mathematics, 60. Published for the Conference Board of the Mathematical Sciences, Washington, DC, 1986.

[9] Rubio de Francia, J. L.: A Littlewood-Paley inequality for arbitrary intervals. Rev. Mat. Iberoamericana 1 (1985), no. 2, 1-14.

[10] Rubio de Francia, J.L.: Martingale and integral transforms of Banach space valued functions. In Probability and Banach spaces (Zaragoza, 1985), 195-222. Lecture Notes in Math., 1221. Springer, Berlin, 1986.

[11] WeIs, L.: Operator-valued Fourier multiplier theorems and maximal $L_{p}$-regularity. Math. Ann. 319 (2001), no. 4, 735-758.

[12] Stein, E. M.: Harmonic analysis: real-variable methods, orthogonality, and oscillatory integrals. Princeton Mathematical Series, 43. Princeton University Press, Princeton, NJ, 1993.

[13] Zygmund, A.: Trigonometric series. Vol. I and II, third edition. Cambridge Mathematical Library, Cambridge University Press, Cambridge, 2002.

Received September 15, 2010.

Denis Potapov: School of Mathematics and Statistics, University of NSW, Kensignton NSW 2052, Australia.

E-mail: d.potapov@unsw.edu.au

Fedor Sukochev: School of Mathematics and Statistics, University of NSW, Kensignton NSW 2052, Australia.

E-mail: f.sukochev@unsw.edu.au

QuAnhua Xu: School of Mathematics and Statistics, Wuhan University, Wuhan 430072, China; and

Laboratoire de Mathématiques, Université de Franche-Comté, 25030 Besançon cedex, France.

E-mail: qxu@univ-fcomte.fr 